\title{
Engineering of Poly(butylcyanoacrylate) Nanoparticles for the Enhancement of the Antitumor Activity of Gemcitabine
}

\author{
José L. Arias, * Visitación Gallardo, and Ma Adolfina Ruiz \\ Department of Pharmacy and Pharmaceutical Technology, Faculty of Pharmacy, \\ University of Granada, Spain
}

\author{
Received June 8, 2009; Revised Manuscript Received August 11, 2009 \\ This paper was retracted on December 16, 2009 (Biomacromolecules DOI: 10.1021/bm9013765).
}

\begin{abstract}
To increase the antitumor activity of gemcitabine along with overcoming its important drawbacks (poor biological half-life and the induction of resistance), we investigated a colloidal carrier based on biodegradable poly(butylcyanoacrylate) nanoparticles. Two gemcitabine loading methods were extensively analyzed: $(i)$ surface adsorption in already formed nanoparticles after incubation in a drug solution; and (ii) gemcitabine addition before the anionic emulsion/polymerization process of the butylcyanoacrylate monomer and subsequent drug trapping into the polymeric network. The latter yielded a higher gemcitabine loading and a sustained release profile. The main factors determining the gemcitabine entrapment into the polymer network and the release kinetic of gemcitabine were analyzed. The cytotoxicity of gemcitabine-loaded polymer was tested in sensitive murine leukemia L1210 wt cells, showing similar activity to the free drug. Following intravenous treatment of mice bearing aggressive leukemia L1210 wt subcutaneous tumors, gemcitabine-loaded poly(butylcyanoacrylate) nanoparticles displayed significantly greater antitumor activity compared to the free chemotherapy agent. This was confirmed by histology studies, suggesting the potential of this gemcitabine delivery system for the efficient treatment of cancer.
\end{abstract}

\section{Introduction}

Despite the long use of numerous multiple drug regimes to improve clinical success, treatment failure frequently occurs even in cancers that are more sensitive to chemotherapy agents. The main reasons for chemotherapy failure are the (i) relatively poor selectivity for target tissues; (ii) large biodistribution and nonintended extravasation with severe side effects in sensitive nontarget tissues; (iii) unfavorable pharmacokinetics (rapid clearance and in vivo degradation) that determine the use of high doses and imposes on patients a rigorous schedule for reaching an antitumor effect; (iv) susceptibility to induce drug resistance; and $(v)$ physicochemical properties of many drugs (e.g., hydrophobicity), which promote the unsuccessful localization at the target site. In addition, the physiology of the tumor is also responsible for chemotherapy failure. The higher hydrostatic pressure inside the tumor induces a pressure gradient that avoids uniform drug diffusion inside the tumor mass. Furthermore, the absence of a functional lymphatic system in these tissues permits the drug to escape from the tumor and its dilution in the surroundings. ${ }^{1-3}$

Gemcitabine ( $2^{\prime}, 2^{\prime}$-difluoro- $2^{\prime}$-deoxycytidine) is a nucleoside analogue of deoxycytidine in which two fluorine atoms are placed in the geminal configuration at the 2'-position of the sugar ring. Gemcitabine is relatively well tolerated when used as a single agent in the treatment of a wide variety of cancers, including lung, colon, head and neck, and ovarian cancers and has also been approved against pancreatic, nonsmall cell lung, bladder, and breast cancer. It requires transportation into the cells by an active transport process before phosphorylation by deoxycytidine kinase into its active form, the 5'-triphosphategemcitabine, which is incorporated into the DNA strand, halting its elongation and causing cell death. The antitumor action involves also the ribonucleotide reductase inhibition.

* To whom correspondence should be addressed. Phone: (+34) 95824 39 00. Fax: (+34) 9582489 58. E-mail: jlarias@ugr.es.
Despite its efficient activity, this compound suffers from various drawbacks, particularly its rapid metabolism following administration in the blood, liver, and kidneys into the inactive derivative $2^{\prime}, 2^{\prime}$-difluoro-2'-deoxyuridine metabolite, which generates the need to use high doses that could be toxic. Hence, gemcitabine has a very short plasma half-life after intravenous (i.v.) administration (8-17 $\mathrm{min}$ in human plasma and $9 \mathrm{~min}$ in murine plasma), which represents a major limitation of this antitumor compound. ${ }^{4-6}$

New therapeutic approaches for cancer treatment have determined the development of polymeric colloids for drug delivery. These nanoplatforms are supposed to obtain a higher antitumor effect with minimal toxicity (thanks to the controlled delivery of the drug to the targeted site and to the decrease in its systemic distribution) and to protect the incorporated chemotherapy agents from in vivo metabolization and elimination (improving their pharmacokinetic profile). Poly(alkylcyanoacrylates) (PACAs) have been extensively investigated for the preparation of this kind of biodegradable drug nanocarriers. ${ }^{7-10}$ In fact, this polymeric family is considered of high interest because of the strong reactivity of the corresponding monomers (able to polymerize easily in water) and due to its stability, biodegradability, biocompatibility, drug compatibility, and targetability. ${ }^{1,9,10}$ Moreover, the toxicity is considered low in the case of multiple dosing (chronic treatment; e.g., $\mathrm{LD}_{50}$ of poly(isobutylcyanoacrylate) in rats: $242 \mathrm{mg} / \mathrm{kg}$ ), as the overloading of cells with slowly degrading polyesters is avoided. ${ }^{11-13}$ In addition, the recovery of cells after the metabolization of the nanoparticles (NPs) takes place easily in vivo due to the considerable low contact time with the degradation products, as they are taken away from their degradation site. ${ }^{10}$ Several clinical trials have also highlighted the good tolerance of these drug carriers. ${ }^{9,14}$

One of the most promising therapeutic applications of PACA NPs is the treatment of both nonresistant and resistant cancers, where they improve the efficiency of the chemotherapy. ${ }^{9,10,15-18}$ 
Their use is justified by the (i) overpowering drug toxicity; (ii) cell death or inhibition of cell growth, a consequence of the toxicity associated with high local concentrations of polymeric degradation products at the tumor cell membrane; and (iii) reversal of the multidrug resistance (MDR) of cancer cells due to both the adsorption of drug-loaded NPs to the cell surface and to the formation, as polymeric degradation occurs, of drug-poly(cyanoacrylic acid) ion pairs that are able to cross the cell membrane without being recognized by the P-glycoprotein $(\mathrm{P}-g p) .9,10,19$

The present study is focused on the preparation of poly(butylcyanoacrylate) (PBCA) NPs loaded with gemcitabine hydrochloride. The parameters determining the amount of drug loaded to the polymer and its release profile are studied. Spectrophotometry was validated and used successfully as the analytical technique in the quantitative determination of both drug loading and release. Gemcitabine loading to PBCA NPs is also qualitatively analyzed by electrophoresis and nuclear magnetic resonance. The antitumor activity of gemcitabine-loaded PBCA NPs in vitro on sensitive murine leukemia L1210 wt cells, as well as in vivo on mice bearing aggressive leukemia L1210 wt subcutaneous tumors is investigated. Cell cycle parameters such as S-phase and apoptosis are also evaluated in vivo.

\section{Materials and Methods}

2.1. Materials. All chemicals used were of analytical quality from Panreac, Spain, except for gemcitabine hydrochloride (Sequoia Research Products Ltd., U.K.); pluronic F-68, formamide, and dextrose (SigmaAldrich Chemical Co., France); and butylcyanoacrylate (a gift from Henkel Loctite, Ireland). Water used in the experiments was deionized and filtered (Milli-Q Academic, Millipore, France).

2.2. Preparation of Poly(butylcyanoacrylate) Nanoparticles. PBCA NPs were synthesized by emulsion/polymerization of the corresponding monomer in an aqueous solution in which the hydroxyl water ions initiate an anionic process of elongation of the polymeric chains. ${ }^{8,10}$ Briefly, the monomer [a $1 \%(\mathrm{w} / \mathrm{v})$ acetonic solution] was added dropwise, under mechanical stirring (1500 rpm), to $25 \mathrm{~mL}$ of an aqueous polymerization medium containing $10^{-4} \mathrm{~N} \mathrm{HCl}$ and the stabilizing agent pluronic F-68 [1\% (w/v)]. After $3 \mathrm{~h}$, the neutralization of the medium was achieved with $25 \mu \mathrm{L}$ of a $\mathrm{NaOH} 10^{-1} \mathrm{M}$ solution to ensure the end of the polymerization. Acetone was then completely evaporated using a Buchi Rotavapor (Switzerland) rotary evaporator to obtain the aqueous suspension of pure PBCA NPs. The whitish suspension was then subjected to a cleaning procedure that included repeated cycles of centrifugation (40 min at $11000 \mathrm{rpm}$, Centrikon T-124 high-speed centrifuge, Kontron, France) and redispersion in water, until the conductivity of the supernatant was $\leq 10 \mu \mathrm{S} / \mathrm{cm}$. The final PBCA NPs aqueous suspensions contained a $5 \%(\mathrm{w} / \mathrm{v})$ of dextrose and a $1 \%$ (w/v) of pluronic F-68.

2.3. Characterization Methods. The size and shape of PBCA NPs were deduced from TEM pictures using a Zeiss EM 902 (Germany) transmission electron microscope set at $80 \mathrm{kV}$ accelerating voltage. Before observation, a dilute suspension of the particles $[\approx 0.1 \%(\mathrm{w} / \mathrm{v})]$ was sonicated for $5 \mathrm{~min}$, and drops of the suspension were placed on copper grids with Formvar film. The grids were then dried at $40{ }^{\circ} \mathrm{C}$ in a convection oven. Mean particle diameters were also determined in triplicate at $25.0 \pm 0.5^{\circ} \mathrm{C}$ by Quasi-Elastic Light Scattering (QELS) using a Nanosizer (Coulter N4MD, Coulter Electronics Inc., U.S.A.). The scattering angle was set at $90^{\circ}$ and the measurement was made after suitable dilution of the aqueous polymer suspensions. The stability of the formulations was evaluated by measuring the size of the particles after 2 weeks of storage at $4.0 \pm 0.5^{\circ} \mathrm{C}$ in water.

To determine the gemcitabine concentration in all the systems investigated, UV absorption measurements were carried out at the maximum absorbance wavelength $(269 \mathrm{~nm})$ in a Perkin-Elmer Lambda
11 UV/vis spectrophotometer (Perkin-Elmer, U.S.A.), using quartz cells of $1 \mathrm{~cm}$ path length. Good linearity was observed at this wavelength between absorbance values and drug concentration up to $0.3 \mathrm{mM}$ in a wide range of $\mathrm{HCl}$ concentrations (from $10^{-2}$ to $10^{-4} \mathrm{M}$ ). The molar absorbance coefficient $\left(8330 \pm 130 \mathrm{~L} \cdot \mathrm{mol}^{-1} \cdot \mathrm{cm}^{-1}\right)$ used in these measurements was calculated from the least-squares fitting of the data obtained in an optical absorbance/gemcitabine concentration calibration curve. No drug solutions above $0.01 \mathrm{M}$ concentration were used as formation of drug crystals was observed. Concentrations above $3 \times$ $10^{-4} \mathrm{M}$ were appropriately diluted to $10^{-4} \mathrm{M}$, as was also carried out in the loading and releasing experiments, to stay in the absorbancegemcitabine concentration linear range. Gemcitabine hydrochloride was found to be stable in $\mathrm{pH} 7.4$ phosphate buffered saline (PBS; BioWhittaker DPBS, Lonza, Belgium), and the validity of the molar absorption coefficient at $269 \mathrm{~nm}$ was confirmed by the analysis of the spectra obtained at this $\mathrm{pH}$.

Before the determination of the amount of drug loaded or released from the PBCA NPs, the spectrophotometric method of analysis was previously validated and verified for accuracy, precision, and linearity. These parameters were determined in standard solutions in six replicates for all the acid $\mathrm{pH}$ range studied and at $\mathrm{pH} 7.4$ (PBS) and also in six calibration standards containing known gemcitabine hydrochloride concentrations added to supernatants of the syntheses performed without drug. The reproducibility of the absorbance spectra of the solutions, during the period of time needed for any of the experiments carried out in this work, was ensured by protection against ambient light, wrapping all glassware with aluminum foil.

2.4. Determination of the Amount of Gemcitabine Loaded to Poly(butylcyanoacrylate) Nanoparticles. Drug loading to a colloidal delivery system can be achieved by following two possible routes: surface adsorption on already formed polymeric particles after incubation in a drug solution, and drug entrapment into the polymeric network by its addition to the polymerization medium or to the corresponding monomer solution before the formation of the NPs. ${ }^{7,8}$

We followed two procedures to determine the gemcitabine surface adsorption on PBCA NPs. One includes the determination of the optical absorbance of supernatants after exposure of the NPs $[\approx 2 \%(\mathrm{w} / \mathrm{v})]$ to gemcitabine hydrochloride solutions of known concentrations for $24 \mathrm{~h}$ under mechanical stirring $(50 \mathrm{rpm})$ at $25.0 \pm 0.5{ }^{\circ} \mathrm{C}$. Gemcitabine adsorption onto PBCA NPs was evaluated by spectrophotometric determinations of the drug remaining in the supernatant solutions, as described below. The supernatants were obtained after a $40 \mathrm{~min}$ centrifugation at $11000 \mathrm{rpm}$. The second one consists of a qualitative estimation of the adsorption process by means of electrophoretic mobility $\left(u_{\mathrm{e}}\right)$ determinations at $25.0 \pm 0.5^{\circ} \mathrm{C}$ after $24 \mathrm{~h}$ of exposure of dilute polymeric suspensions $[\approx 0.1 \%(\mathrm{w} / \mathrm{v})]$ to different drug concentrations at this temperature under mechanical stirring (50 rpm), using a Zetasizer 2000 electrophoresis device (Malvern Instruments Ltd., U.K.). Furthermore, to analyze the effect of ionic strength variations, the experiments were performed both with and without $10^{-3} \mathrm{M} \mathrm{NaCl}$ in solution. In both procedures, all solutions contained $1 \%(\mathrm{w} / \mathrm{v})$ pluronic F-68 and 5\% (w/v) dextrose. All the experiments were carried out in triplicate.

Gemcitabine absorption measurements were carried out in triplicate by application of Beer's law to the optical absorbance of aqueous solutions in which the synthesis of the PBCA NPs was carried out. For the method to be accurate, we considered the contribution to the absorbance of sources other than variations in drug concentration when evaluating the gemcitabine incorporation: mainly the products of the polymer degradation in the medium, but also the surfactant agent pluronic F-68 and the electrolytes that can be presented in the medium. Hence, spectrophotometric determinations of the drug remaining in the supernatant solutions (as compared to the initial amount) helped us in the estimation of the gemcitabine loaded: ${ }^{7,8,20-23}$ the amount of drug present in the aqueous solution after the absorption process was obtained from the absorbance of the solution at $269 \mathrm{~nm}$ after subtracting the absorbance of the supernatant produced in the same conditions but 
without gemcitabine in solution. Briefly, the drug loading procedure includes: $(i)$ preparation of the aqueous polymerization medium (25 $\mathrm{mL}$ ) with the appropriate amounts of gemcitabine hydrochloride, $\mathrm{HCl}$ and pluronic F-68; (ii) dropwise addition of an acetonic solution of the butylcyanoacrylate monomer $[1 \%(w / v)]$ mixed with adequate quantities of a methanolic gemcitabine solution; (iii) mechanical stirring of the medium (1500 rpm) for $3 \mathrm{~h}$; (iv) stopping the reaction by addition of $25 \mu \mathrm{L}$ of a NaOH $10^{-1} \mathrm{M}$ solution to ensure the total monomer consumption; $(v)$ complete evaporation of acetone and methanol by using a Rotavapor; (vi) centrifugation of the solids (11000 rpm for 40 min); and (vii) determination of the drug loaded by measuring its concentration in the supernatant. We did not investigate the influence of the monomer concentration on gemcitabine absorption as no important effect on drug loading was previously reported. ${ }^{7,24,25}$ Gemcitabine incorporation to PBCA NPs was expressed in terms of gemcitabine entrapment efficiency (\%) [(encapsulated gemcitabine (mg)/ total gemcitabine in the colloidal suspension $(\mathrm{mg})) \times 100]$ and gemcitabine loading $(\%)$ [(encapsulated gemcitabine $(\mathrm{mg}) / \mathrm{PBCA}$ carrier $(\mathrm{mg})) \times 100] .{ }^{26}$

Gemcitabine absorption into PBCA NPs was also qualitatively checked by ${ }^{1} \mathrm{H}$ nuclear magnetic resonance $\left({ }^{1} \mathrm{H}\right.$ NMR). ${ }^{1} \mathrm{H}$ NMR analyses (Bruker AM-300, U.S.A.) were obtained at $25.0 \pm 0.5{ }^{\circ} \mathrm{C}$ using deuterated dimethyl sulfoxide (DMSO- $d_{6}$ ) as solvent. The data was used for the chemical characterization of gemcitabine, PBCA, and gemcitabine-loaded PBCA NPs. ${ }^{27-29}$

2.5. In Vitro Gemcitabine Release from Poly(butylcyanoacrylate) Nanoparticles. Drug release determinations from PBCA NPs loaded with gemcitabine after an adsorption process or after drug incorporation into the polymeric network were carried out in triplicate following the dialysis bag method. PBCA NPs were prepared using a $10^{-2} \mathrm{M}$ gemcitabine concentration in the adsorption/absorption medium. PBS ( $\mathrm{pH}=7.4 \pm 0.1)$ or a $\mathrm{HCl}$ solution $(\mathrm{pH}=2.0 \pm 0.1)$ was used as the release medium maintained at $37.0 \pm 0.5{ }^{\circ} \mathrm{C}$ during all the experiments.

Briefly, the bags were soaked in water for $12 \mathrm{~h}$ before use. The dialysis bag retains the PBCA NPs and allows the free gemcitabine to come into the dissolution media with a cutoff of $2000 \mathrm{Da}$ (Spectrum Spectra/Por 6 dialysis membrane tubing, U.S.A.). A total of $2 \mathrm{~mL}$ of gemcitabine-loaded PBCA NPs suspension (containing $2 \mathrm{mg} / \mathrm{mL}$ of gemcitabine) was poured into the bag with the two ends fixed by clamps. The bags were placed in a conical flask filled with $100 \mathrm{~mL}$ of the receiving phase and were stirred at $200 \mathrm{rpm}$. At prefixed times $(0.08$, $0.25,0.5,0.75,1,2,3,4,5,6,9,12$, and $24 \mathrm{~h}), 5 \mathrm{~mL}$ samples of the medium were withdrawn for UV-vis spectrophotometric analysis at $269 \mathrm{~nm}$. An equal volume of PBS or $\mathrm{HCl}$ solution, maintained at the same temperature, was added after sampling to ensure sink conditions. The same measurement procedure followed in the estimation of drug loading was followed.

2.6. Cytotoxicity Assay on L1210 wt Cells. L1210 wt murine leukemia cells were cultured in RPMI 1640 supplemented with $10 \%$ fetal calf serum, $50 \mathrm{U} / \mathrm{mL}$ penicillin, $50 \mu \mathrm{g} / \mathrm{mL}$ streptomycin, and 2 $\mathrm{mM}$ L-glutamine. The antitumor activity of gemcitabine and gemcitabine-loaded PBCA NPs toward this cell line was determined using the 3-[4,5-dimethylthiazol-2-yl]-3,5-diphenyl tetrazolium bromide (MTT) test, measuring mitochondrial dehydrogenase activity. Blank PBCA NPs served as placebo control. The cells in exponential growth phase were seeded into 96-well plates and were preincubated for $24 \mathrm{~h}$ at $37.0 \pm$ $0.5{ }^{\circ} \mathrm{C}$ in a humidified atmosphere of $5 \% \mathrm{CO}_{2}$ in air. Different concentrations of gemcitabine, gemcitabine-loaded PBCA NPs, and drug-unloaded (blank) PBCA NPs (at dilutions similar to that of the drug-loaded NPs) were added to the cells in the culture medium. Each concentration was tested in triplicate. After $72 \mathrm{~h}$ at $37.0 \pm 0.5^{\circ} \mathrm{C}, 200$ $\mu \mathrm{L}$ of MTT solution in cell culture medium $(0.5 \mathrm{mg} / \mathrm{mL})$ was added to each well. After incubation for $2.5 \mathrm{~h}$ at $37.0 \pm 0.5{ }^{\circ} \mathrm{C}$, the culture medium was removed and the resultant formazan crystals were dissolved in $200 \mu \mathrm{L}$ of dimethyl sulfoxide. The absorbance of converted dye, which is proportional to the number of viable cells, was measured at $570 \mathrm{~nm}$ using a microplate reader (Metertech $\Sigma 960$, Fisher Bioblock, Illkirch, France). The percentage of surviving cells was calculated as the absorbance ratio of treated to untreated cells.

2.7. In Vivo Anticancer Activity of Gemcitabine and Gemcitabine-Loaded Poly(butylcyanoacrylate). The animal experiments were carried out according to the principles of laboratory animal care and legislation in force in Spain. DBA/2 mice (5 weeks old) weighing $\approx$ 15-18 g were used. The mice were provided with standard mouse food and water ad libitum. L1210 wt murine subcutaneous tumor model was developed by subcutaneous injection of the exponentially growing L1210 tumor cells $\left(10^{6}\right.$ cells) in suspension containing 30\% growth factor reduced Matrigel, above the right hind leg portion of the mice. After waiting for 5 days, when the mice developed palpable tumors at the injection site, the mice were divided into four groups of 6 each, that is, untreated, treated with blank PBCA NPs (placebo), treated with gemcitabine $20 \mathrm{mg} / \mathrm{kg}$, and treated with gemcitabine-loaded PBCA NPs equivalent to $20 \mathrm{mg} / \mathrm{kg}$ gemcitabine. All the groups of tumor bearing mice, except untreated, were intravenously treated on days $0,3,7$, and 10. The mice were monitored regularly for differences in tumor volume to assess the antitumor efficacy.

2.8. Tumor Histology Studies. Determination of S-Phase of Cell Cycle and Apoptosis. After the completion of the treatment schedule, one mouse from each group was sacrificed and the tumor was isolated and fixed overnight in Finefix solution at $25.0 \pm 0.5^{\circ} \mathrm{C}$. Then the tumors were sequentially treated with ethanol and toluene and fixed in paraffin. Finally, $3 \mu \mathrm{m}$ sized paraffin embedded tumor sections fixed on glass slide for overnight at $56.0 \pm 0.5^{\circ} \mathrm{C}$ in an oven were cut using a microtome blade. The tumor sections were analyzed for histology by optical microscopy using the Hematoxylin-EosinSaffran (HES) staining technique. Briefly, paraffin embedded tumor sections were deparaffinated by successive treatment with toluene and ethanol and finally washed with purified water. Then the tumor sections were stained using hematoxylin, eosin, and saffron by successive washings in water or ethanol and finally with toluene. Afterward, the stained tumor sections were mounted for observation under $40 \times$ magnification using an optical microscope.

To further investigate the mechanism behind the superiority of the antitumor activity of gemcitabine-loaded PBCA NPs compared to gemcitabine, three days following the i.v. injection of either free gemcitabine $(20 \mathrm{mg} / \mathrm{kg})$ or gemcitabine-loaded PBCA NPs $(20 \mathrm{mg} / \mathrm{kg}$ equiv), tumor cells were collected and studied for S-phase and apoptosis by measuring the sub- $\mathrm{G}_{0} / \mathrm{G}_{1}$ population. Cell cycle events and apoptosis were determined by flow-cytometric measurements of cellular DNA content using the DNA-intercalating fluorochrome, propidium iodide (PI). Briefly, tumor cells were washed once with $2 \mathrm{~mL}$ of PBS. $10^{6}$ cells were fixed overnight in $70 \%$ ethanol. Then the cells were centrifuged to remove ethanol, washed with PBS, and treated with extraction buffer (a mixture of 192 parts of $0.2 \mathrm{M}$ disodium hydrogen phosphate and 8 parts of $0.1 \mathrm{M}$ citric acid). Cells were then washed with $2 \mathrm{~mL}$ PBS and treated with ribonuclease-A $(200 \mu \mathrm{g} / \mathrm{mL})$ for 30 $\min$ at $37.0 \pm 0.5^{\circ} \mathrm{C}$. Subsequently, cells were stained with PI (50 $\mu \mathrm{g} / \mathrm{mL}$ ) in PBS. Measurements were made with a laser-based (488 nm) flow cytometer (FACS Calibur, Beckton and Dickenson, U.S.A.) and data acquired using the Cell Quest software (Beckton and Dickenson, U.S.A.). Off-line gating was performed using appropriate windows created by analysis of light scattering from untreated cells.

2.9. Statistical Analysis. Statistical analysis was performed by the use of the Student's $t$-test. Values with $p<0.05$ and $p<0.01$ were considered as significantly different.

\section{Results and Discussion}

3.1. Particle Size and Morphology. The polymerization process is governed by the relative amounts of the $\mathrm{OH}$ groups of the polyhydroxylic surfactant (pluronic F-68) and the $\mathrm{OH}^{-}$ ions from water molecules, which are weak initiators of the polymerization. Equilibrium must be reached between initiating 


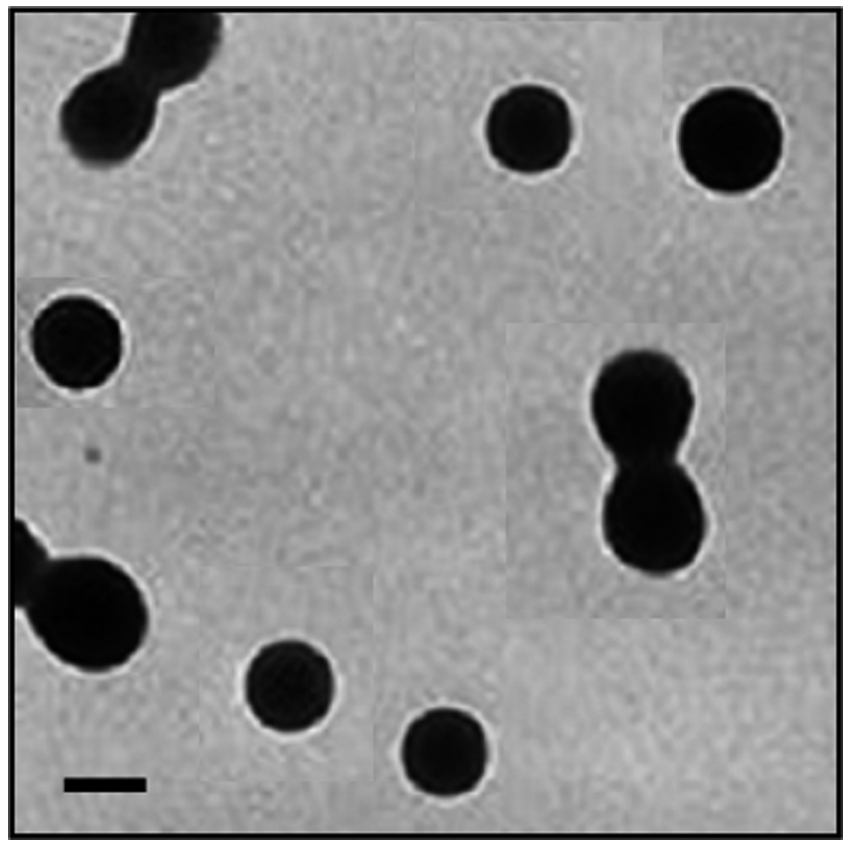

Figure 1. Transmission electron microscopy picture of PBCA NPs. Bar length: $150 \mathrm{~nm}$.

and terminating agents to obtain polymer suspensions free of macroaggregates or bulky sediments and with a suitable particle size for drug delivery. The polymerization rate is governed by this relationship and falls as the acidity of the medium increases. An inadequate polymerization rate will yield a large proportion of bulk polymer not in the form of particles; therefore, this reaction must be slow enough to give homogeneously milky suspensions, with negligible formation of polymer residues, but not so slow as to yield excessive particle coagulation. ${ }^{8}$

The emulsion/polymerization technique for the synthesis of PBCA NPs allowed, in agreement with previous studies, $7,8,30$ the formation of well-stabilized spherical NPs with a narrow size distribution in the colloidal size range and suitable for parenteral administration [average diameter \pm standard deviation, and polydispersity index: $170 \pm 60 \mathrm{~nm}$ and 0.094 , respectively (Figure 1)]. No presence of aggregates or bulky sediments was observed. The size of the PBCA particles and the quality of the suspensions did not vary significantly when loaded with different drug amounts, as previously observed. ${ }^{8,31}$ Furthermore, after 2 weeks of storage at $4.0 \pm 0.5^{\circ} \mathrm{C}$ in water, no appreciable change in the size of the NPs was detected by QELS and no gemcitabine precipitation or NP aggregation was observed.

3.2. Gemcitabine Surface Adsorption onto Poly(butylcyanoacrylate) Nanoparticles. A positive effect of the drug concentration in the contact medium on the adsorption efficiency onto PBCA NPs was observed (Figure 2): the entrapment efficiency of gemcitabine increases with the amount of drug in solution up to $\approx 32 \%$ for the concentrations investigated. However, these results can be considered low (drug loading $\approx$ $5 \%$ ). This low gemcitabine surface adsorption to the hydrophobic $\mathrm{PBCA}^{32}$ can be explained if we consider the hydrophilic nature of this drug. In fact, the logarithm of the distribution coefficient of this drug at $\mathrm{pH} 7.4$ in the system $n$-octanol/water was determined to be $\log D_{\text {oct/water }}=-1.24 .^{33}$ Thus, the approach of this nucleoside analogue from the aqueous phase to the hydrophobic NP surface is unfavored.

The electrophoretic mobility $\left(u_{\mathrm{e}}\right)$ determinations qualitatively confirmed these conclusions. Like other electrokinetic tech-
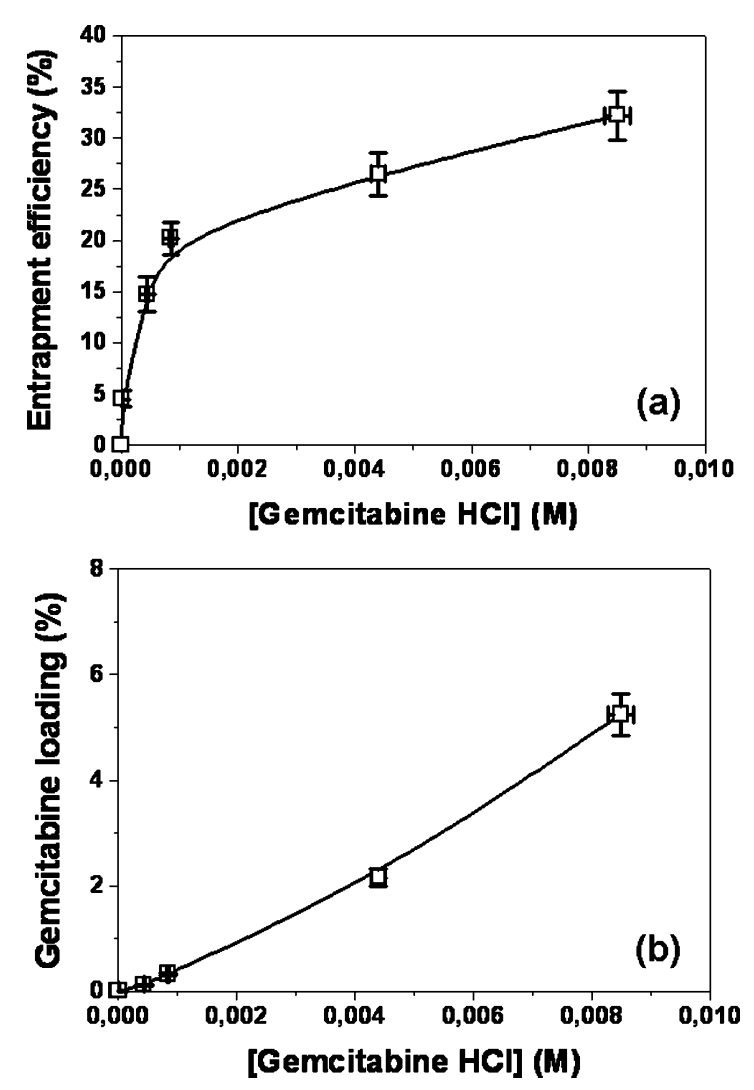

Figure 2. Gemcitabine entrapment efficiency (\%; a) and gemcitabine loading (\%; b) of PBCA NPs as a function of the equilibrium drug concentration. The lines are guides to the eye.

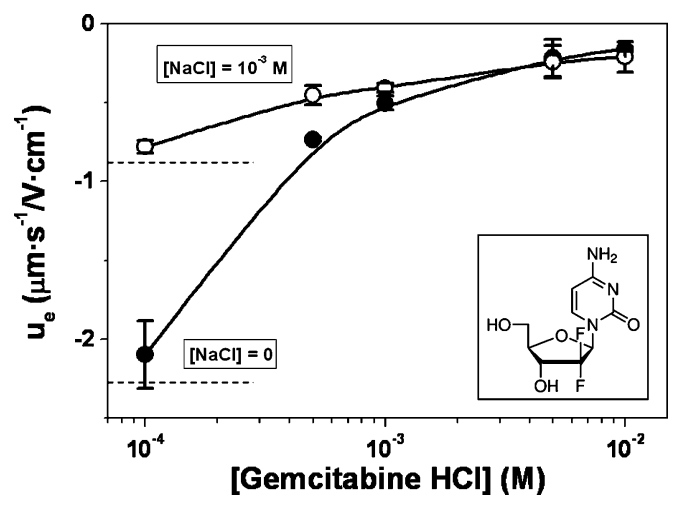

Figure 3. Electrophoretic mobility $\left(u_{\mathrm{e}}\right)$ of PBCA NPs $(\bullet, 0)$ as a function of gemcitabine concentration, in the presence (open symbols) and absence (full symbols) of $10^{-3} \mathrm{M} \mathrm{NaCl}$ (inset: chemical structure of gemcitabine). The lines are guides to the eye. The dashed lines indicate the mean $u_{\mathrm{e}}$ value of PBCA in the absence of this drug for $1 \mathrm{mM} \mathrm{NaCl}$ (upper line) and in water (lower line).

niques, electrophoresis is very sensitive to surface modification via adsorption of charged entities, even at rather small amounts. Thus, $u_{\mathrm{e}}$ determinations can be used to qualitatively confirm drug adsorption and even suggest the possible role of electrostatic interactions in this process. ${ }^{7,32}$ Data corresponding to the PBCA NPs in gemcitabine solutions are shown in Figure 3: $u_{\mathrm{e}}$ displays a general trend to rise (toward progressively less negative values, mainly in the absence of $\mathrm{NaCl}$ ) as the drug concentration is increased. In this figure we have included the $u_{\mathrm{e}}$ values of the PBCA NPs in the absence of drug: note that it is negative for both $1 \mathrm{mM} \mathrm{NaCl}$ concentration and pure water. $^{27,33,34}$ This net negative surface charge must stem from weak acrylic acid groups of the polymer. Moreover, the negative charge is also a consequence of strong groups corresponding 
This paper was retracted on December 16, 2009 (Biomacromolecules DOI: 10.1021/bm9013765).
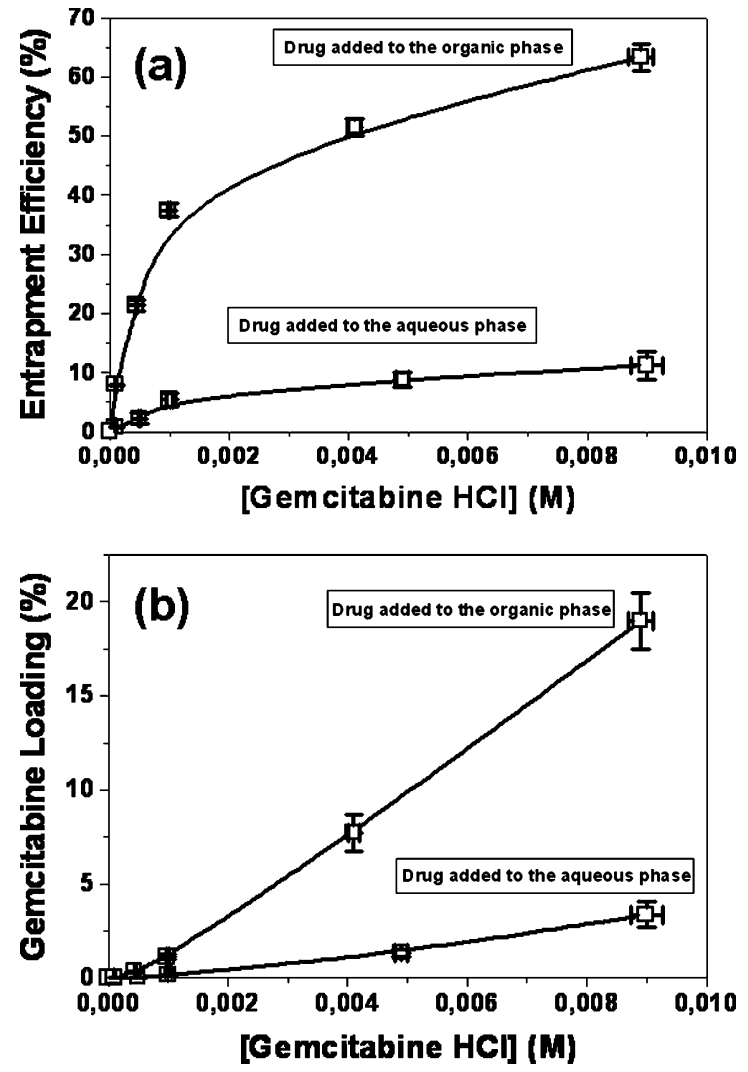

Figure 4. Gemcitabine entrapment efficiency (\%; a) and gemcitabine loading $(\% ; b)$ of PBCA NPs as a function of the equilibrium drug concentration and as a function of the preparation procedure [drug incorporated into either the aqueous polymerization medium (drug added to the aqueous phase) or into the acetonic monomer solution (drug added to the organic phase)]. The lines are guides to the eye.

to dissociated end molecules of pluronic F-68 that remain adsorbed onto the NP surface, even after the preparation procedure followed for the polymer samples. This contribution has also been reported when dextran-70 is used as stabilizer of this polymer. ${ }^{7}$ In any case, electrostatic attraction can be claimed to be an essential mechanism for gemcitabine incorporation to the surface of PBCA NPs, so that the originally negative charge is reduced by the electrostatically favored adsorption of drug (positively charged species, presumably coming from the protonation of the $-\mathrm{NH}_{2}$ group of the drug molecule). This also explains the $\mathrm{NaCl}$ effect: the presence of this indifferent electrolyte yields a mobility reduction because of the double layer compression. In addition, $\mathrm{NaCl}$ screens the attraction between drug molecules and polymer NPs thus leading to a reduction of $u_{\mathrm{e}}$ when the gemcitabine concentration increases, that is more significant when there is no $\mathrm{NaCl}$ in the medium. ${ }^{7,8}$

3.3. Modulation of the Gemcitabine Absorption to Poly(butylcyanoacrylate) Nanoparticles by the Polymerization Conditions. 3.3.1. Influence of the Preparation Procedure and the Gemcitabine Concentration. Because of the hydrophilic nature of gemcitabine, its approach from the aqueous polymerization phase to the hydrophobic matrix of PBCA NPs is expected to be unfavored. ${ }^{33}$ Therefore, we tried to set the polymerization conditions that minimize the escape of this chemotherapy agent from a mechanical trapping within the PBCA matrix. With this aim, we first studied the influence of placing the drug into either the aqueous polymerization medium (drug added to the aqueous phase) or into the acetonic monomer solution (drug added to the organic phase) on the gemcitabine
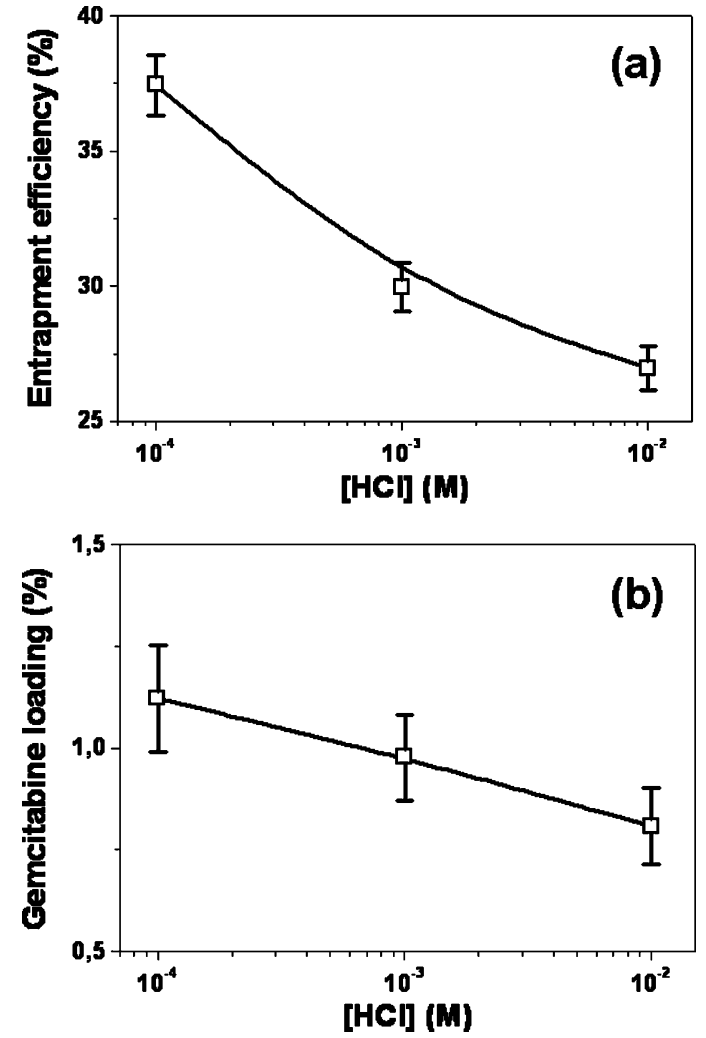

Figure 5. Gemcitabine entrapment efficiency (\%; a) and gemcitabine loading $(\% ; b)$ of PBCA NPs as a function of the $\mathrm{HCl}$ molar concentration. The lines are guides to the eye.

absorption process. Briefly, the absorption procedure involved follows: ( $i$ ) the preparation of the aqueous polymerization medium [1\% (w/v) pluronic F-68 and $0.1 \mathrm{mM} \mathrm{HCl}]$ with the appropriate amounts of drug, and the subsequent dropwise addition of an acetonic solution of the butylcyanoacrylate monomer $[1 \%(\mathrm{w} / \mathrm{v})]$; or $(\mathrm{ii})$ the preparation of the aqueous polymerization medium [1\% (w/v) pluronic F-68 and $0.1 \mathrm{mM}$ $\mathrm{HCl}]$ and the subsequent dropwise addition of an acetonic solution of the butylcyanoacrylate monomer [1\% (w/v)] containing adequate quantities of a methanolic gemcitabine solution. All other steps previously described for the NP preparation were kept constant (sections 2.2. and 2.4.). Figure 4 shows the amount of gemcitabine absorbed by PBCA NPs as a function of the equilibrium drug concentration: both the entrapment efficiency $(\%)$ and the drug loading (\%) were significantly enhanced when gemcitabine was added into the acetonic solution of the butylcyanoacrylate monomer, whatever the initial drug concentration fixed. For instance, when the initial gemcitabine concentration was $10^{-2} \mathrm{M}$, these parameters rise from $11.2 \pm 2.3 \%$ and $3.4 \pm 0.7 \%$ (when the drug is added to the aqueous phase) to $63.3 \pm 2.3 \%$ and $18.9 \pm 1.5 \%$ (when the drug is added to the organic phase), respectively. This is probably due to the difficulty of gemcitabine to escape from the polymeric network when it is embedded in it. This was not observed when this hydrophilic compound forms part of the aqueous polymerization phase. ${ }^{7,8}$ With respect to the initial gemcitabine concentration, a positive effect of the increment in drug concentration in solution was observed on the entrapment efficiency, as has been reported with other drugs, ${ }^{7,8}$ suggesting a trend toward saturation for the maximum concentrations investigated. Drug loading (\%) was also increased for the gemcitabine concentrations studied.

3.3.2. Effect of $\mathrm{HCl}$ Concentration. As mentioned above, because gemcitabine is essentially hydrophilic, a fast polymerization will mechanically entrap as much chemotherapy agent 
This paper was retracted on December 16, 2009 (Biomacromolecules DOI: 10.1021/bm9013765).

F Biomacromolecules, Vol. $x x x$, No. $x x, X X X X$

Arias et al.



Figure 6. ${ }^{1} \mathrm{H}$ NMR spectra of (a) blank PBCA NPs, (b) gemcitabine, and (c) gemcitabine-loaded PBCA NPs.

as possible; however, it should also be considered that a large amount of bulky polymer solids or macroaggregates will also be generated by a very fast process. Polymerization of alkylcyanoacrylates is an anionic process initiated by covalent bases, and hence, the kinetics are governed by the relative amounts of the alcoholic $-\mathrm{OH}$ groups of the surfactant (in our case pluronic F-68) and $\mathrm{OH}^{-}$ions coming from water dissociation. As the acidity of the medium decreases, the polymerization rate increases; as a result of this, it can be concluded that the $\left[\mathrm{H}^{+}\right]$ will determine both the polymerization rate and the drug absorption. ${ }^{7,8}$ Hence, we investigated gemcitabine absorption under $\mathrm{HCl}$ concentrations ranging from $10^{-4}$ to $10^{-2} \mathrm{~N}$ in the following conditions: $10^{-3} \mathrm{M}$ gemcitabine hydrochloride, $1 \%$ (w/v) pluronic F-68, 1\% (w/v) monomer, drug added to the organic phase, and $3 \mathrm{~h}$ of polymerization. Figure 5 shows that gemcitabine absorption is clearly affected by the $\left[\mathrm{H}^{+}\right]$of the polymerization medium and that it is largest at $10^{-4} \mathrm{~N} \mathrm{HCl}$ $(\mathrm{pH} \approx 4)$ : as the $\mathrm{OH}^{-}$concentration decreases, the polymerization rate becomes slower and the absorption falls. If we compare the results obtained at $10^{-4} \mathrm{~N} \mathrm{HCl}$ and at $10^{-2} \mathrm{~N} \mathrm{HCl}$, it is clear that the entrapment efficiency (\%) falls from $37.4 \pm$ $1.1 \%$ to $26.9 \pm 0.8 \%$. A slight decrease is also observed in drug loading (\%) values: from $1.1 \pm 0.1 \%$ to $0.8 \pm 0.1 \%$. Finally, although a higher absorption is expected under more basic conditions, syntheses carried out at $[\mathrm{HCl}]<10^{-4} \mathrm{~N}$ yielded particles unsuitable for parenteral administration.

3.3.3. Effect of Surfactant Concentration. In this paragraph we describe our data on the role of changes in pluronic F-68 
This paper was retracted on December 16, 2009 (Biomacromolecules DOI: 10.1021/bm9013765).

concentration [from 0 to $2 \%(\mathrm{w} / \mathrm{v})$ ] on drug loading, keeping all other parameters of the synthesis constant $[1 \%(\mathrm{w} / \mathrm{v})$ butylcyanoacrylate concentration, $10^{-3} \mathrm{M}$ gemcitabine, $0.1 \mathrm{mM}$ $\mathrm{HCl}$, drug added to the organic phase, and $3 \mathrm{~h}$ of polymerization]. No significant influence on gemcitabine absorption was found as the surfactant concentration rises. However, its role in the generation of polymer NPs is crucial due to the combination of its initiating and stabilizing actions close to other frequently used agents, such as polyethylene glycol or dextran$70 .{ }^{7}$ If pluronic F-68 concentrations $<1 \%(\mathrm{w} / \mathrm{v}$ ) are used, a fast coagulation of the suspensions is observed, and the formation of macroaggregates and large solid particles takes place. However, pluronic F-68 concentrations $\geq 1 \%$ (w/v) yield very stable whitish dispersions where sedimented macroaggregates are not observable. Therefore, it was concluded that the addition of this stabilizing agent to the polymerization medium generates homogeneous distributions of PBCA NPs with reduced size and great uniformity, without negatively affecting the drug entrapment efficiency and drug loading.

3.3.4. Qualitative Evaluation of the Gemcitabine Absorption to Poly(butylcyanoacrylate) Nanoparticles. Figure 6 shows the ${ }^{1} \mathrm{H}$ NMR spectra of blank PBCA NPs, gemcitabine hydrochloride, and gemcitabine-loaded PBCA NPs. The most significant bands of both the chemotherapy agent and the polymer were identified by comparison with previous data. ${ }^{27-29}$ The main feature of this figure is the presence of the bands of gemcitabine in the spectrum of the drug-loaded PBCA NPs, which clearly demonstrates that gemcitabine was absorbed into this polymeric matrix.

3.4. Study of the In Vitro Gemcitabine Release from Poly(butylcyanoacrylate) Nanoparticles. Gemcitabine-loaded PBCA NPs used in the in vitro drug release experiments were the ones obtained under the best gemcitabine-loading conditions: preparation of the aqueous polymerization medium $[1 \%(\mathrm{w} / \mathrm{v})$ pluronic F-68 and $0.1 \mathrm{mM} \mathrm{HCl}$ and subsequent dropwise addition of an acetonic solution of the butylcyanoacrylate monomer $[1 \%(\mathrm{w} / \mathrm{v})]$ mixed with a methanolic gemcitabine solution $\left(10^{-2} \mathrm{M}\right)$. All other steps previously detailed in sections 2.2. and 2.4. for the preparation of these NPs were kept constant. See section 3.3.1. to check the amount of drug absorbed under these conditions. Figure $7 \mathrm{a}$ shows gemcitabine release from PBCA NPs as a function of time in PBS $(\mathrm{pH} 7.4 \pm 0.1)$ at 37.0 $\pm 0.5^{\circ} \mathrm{C}$. A biphasic process occurs: first, an early rapid release up to $\approx 43 \%$ took place within $1 \mathrm{~h}$, while the remaining gemcitabine absorbed was slowly liberated during the next $11 \mathrm{~h}$. The rapid release probably represents the loss of surfaceassociated and poorly entrapped (adsorbed on the surface pores) drug. Gemcitabine release during the slower release phase may result from particle disintegration by surface erosion, from drug diffusion through the polymeric matrix, or both. Such a biphasic profile, typical of the PACA family, suggests that the major fraction of gemcitabine was entrapped into the polymeric network rather than adsorbed onto the NP surface. ${ }^{7,8,31}$ In the case of the release of the adsorbed drug, gemcitabine-loaded PBCA NPs used in the in vitro release experiments were the ones obtained when the adsorption medium had a gemcitabine concentration of $10^{-2} \mathrm{M}$. See section 3.2. to check the amount of drug loaded under these conditions. The release of the adsorbed gemcitabine was almost complete after $1 \mathrm{~h}$. This rapid release suggests that the drug was likely adsorbed only on the external NP surface and could be a consequence of the weak physisorption of this hydrophilic drug with the hydrophobic PBCA surfaces.
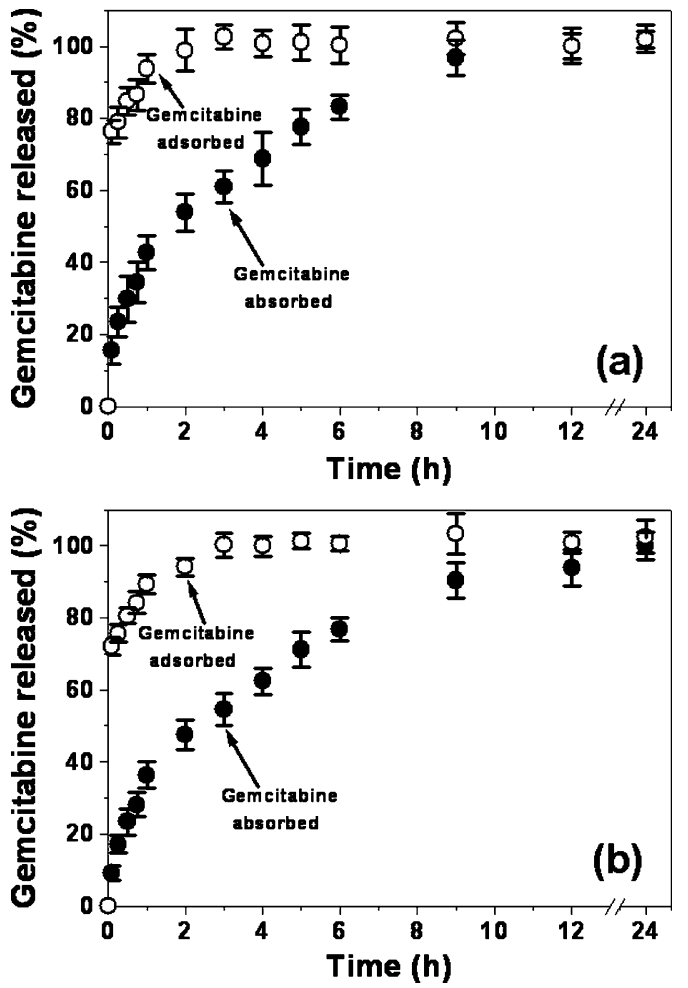

Figure 7. Release of gemcitabine, adsorbed (open symbols: $O$ ) or absorbed (full symbols: -) from PBCA NPs as a function of the incubation time in (a) PBS $(\mathrm{pH}=7.4 \pm 0.1)$ and (b) $\mathrm{HCl}$ solution $(\mathrm{pH}$ $=2.0 \pm 0.1)$, at $37.0 \pm 0.5^{\circ} \mathrm{C}$.

When the $\mathrm{pH}$ of the medium was fixed to 2 , the gemcitabine release profiles (Figure $7 \mathrm{~b}$ ) were similar to those obtained at $\mathrm{pH}=7.4$ (Figure 7a), except that a slower release rate was observed: $\approx 36 \%$ within $1 \mathrm{~h}$, while the remaining drug absorbed was slowly released during the next $23 \mathrm{~h}$. The significant acceleration of the surface erosion rate as the $\mathrm{pH}$ increased is a consequence of the key role played by the $\mathrm{OH}^{-}$ions on the hydrolysis of the alkyl side chain: the increase in $\left[\mathrm{OH}^{-}\right]$with increasing $\mathrm{pH}$ induces a higher hydrolytic attack on the polymer $\mathrm{C}-\mathrm{C}$ bonds, resulting in a faster degradation and, as a consequence, in a more rapid drug release. ${ }^{8,10,35}$ All other characteristics of this biphasic process, pointed out before in relation to data in PBS, remained essentially unchanged. Thus,

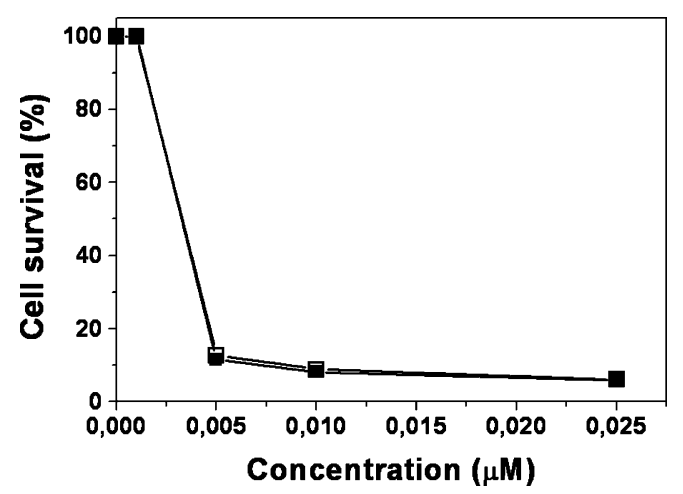

Figure 8. In vitro cytotoxicity on L1210 wt murine leukemia cells of free gemcitabine (ם) and gemcitabine-loaded PBCA NPs ( $\square$ ). The values are the mean \pm standard deviation (S.D.) of three determinations. The SD bars plotted for the curves do not appear in the figure due to the very low SD values. Blank PBCA NPs at dilutions corresponding to that of gemcitabine-loaded PBCA NPs were used as control and did not show any cytotoxicity at the dilutions tested. 


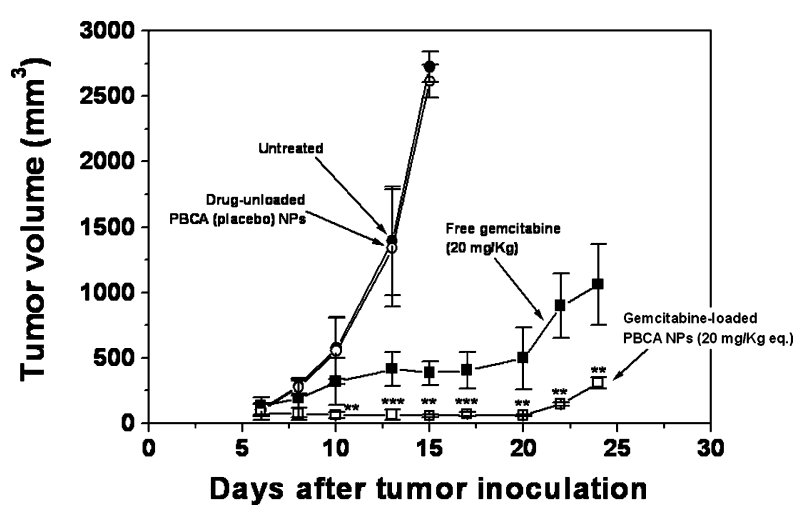

Figure 9. Antitumor activity of gemcitabine-loaded PBCA NPs (20 $\mathrm{mg} / \mathrm{kg}$ equiv; $\square$ ) compared with untreated $(\bullet)$, drug-unloaded PBCA (placebo) NPs (polymer concentration similar to that injected in case of gemcitabine-loaded PBCA NPs; O) and free gemcitabine (20 mg/ $\mathrm{kg}$; ), administered intravenously on days $0,3,7$, and 10 following the development of palpable tumors into L1210 wt subcutaneous tumor bearing mice. The values shown in the graph are the mean \pm SD of 6 mice. Statistical analysis was performed by student's $t$-test $\left({ }^{* *} p<0.05,{ }^{* * *} p<0.001\right)$.

gemcitabine release from PBCA loaded by an adsorption process is faster and, in fact, can be considered complete after $3 \mathrm{~h}$.

3.5. Cytotoxicity of Gemcitabine-Loaded Poly(butylcyanoacrylate) Nanoparticles. The cytotoxicity of gemcitabineloaded PBCA NPs against L1210 wt leukemia cells [50\% inhibitory concentration $\left(\mathrm{IC}_{50}\right)$ value of $3.29 \mathrm{nM}$ ] was similar to that of gemcitabine free $\left(\mathrm{IC}_{50}=3.24 \mathrm{nM}\right.$; Figure 8). These results suggest that the drug-loaded NPs are as efficient as free gemcitabine on this tumor cell line.

3.6. Antitumor Activity of Gemcitabine and GemcitabineLoaded Poly(butylcyanoacrylate) Nanoparticles. The antitumor activity of gemcitabine-loaded PBCA NPs was compared with free gemcitabine and drug-unloaded PBCA (placebo) NPs on L1210 wt subcutaneous tumor bearing mice (Figure 9). It is clear from this figure that placebo NPs were inactive, resulting in tumor progression similar to that of the untreated group. Gemcitabine treatment led to a considerable control over tumor progression as compared with placebo and untreated groups. However, gemcitabine-loaded PBCA NPs caused first a rapid and dramatic tumor regression until day 20 with further significant control of tumor progression as compared with gemcitabine treatment $(p<0.05$ and $p<0.01)$. This clearly suggests its superior antitumor activity over free gemcitabine. This is likely due to the protection of gemcitabine from rapid metabolism generally occurring soon after i.v. injection due to the deamination action by deoxycytidine deaminase ${ }^{5}$ and, additionally, due to the improved tumor accumulation of this drug delivery system probably due to the enhanced permeability and retention (EPR) effect owing to their very small particle size, followed by a gemcitabine controlled release that leads to a longer exposure of the tumor cells to the chemotherapy agent. ${ }^{9,10}$

At the end of the treatment schedule, tumors were isolated from one mouse from each of the treated groups and processed for histological observation using the Hematoxylin-EosinSaffran (HES) staining technique. Untreated and placebo tumor sections showed a highly dense cancerous tissue completely covered by the tumor cells (Figure 10). Gemcitabine-treated tumor sections showed less cancer cell density as compared to untreated tumors, while the gemcitabine-loaded PBCA NPs tumor sections showed elimination of the majority of the tumor cells, displaying a normal tissue background in the sections.

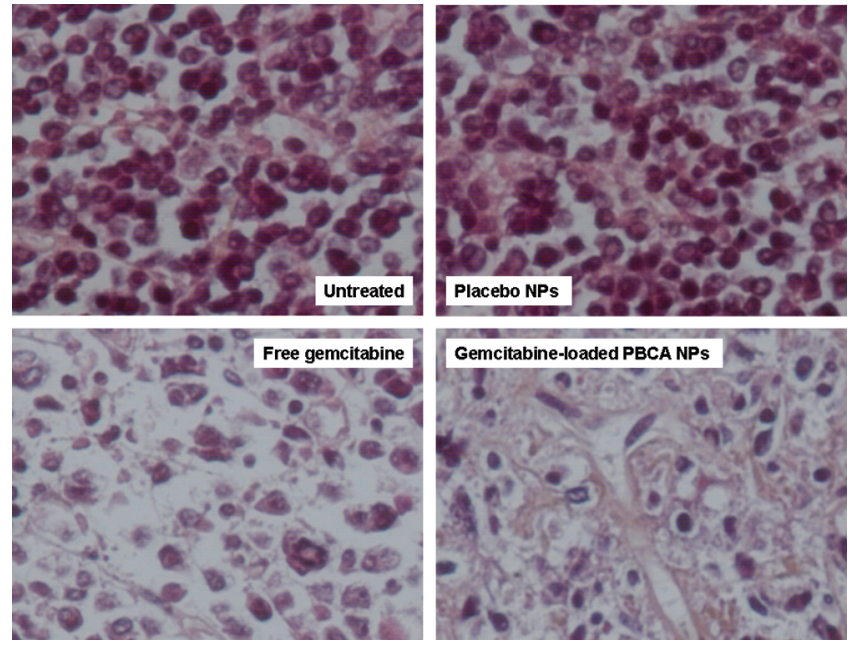

Figure 10. Histological evaluation of tumors isolated from mice, untreated or after treatment with drug-unloaded PBCA (placebo) NPs (polymer concentration similar to that injected in case of gemcitabineloaded PBCA NPs), free gemcitabine $(20 \mathrm{mg} / \mathrm{kg})$, or gemcitabineloaded PBCA NPs (20 mg/kg equiv).
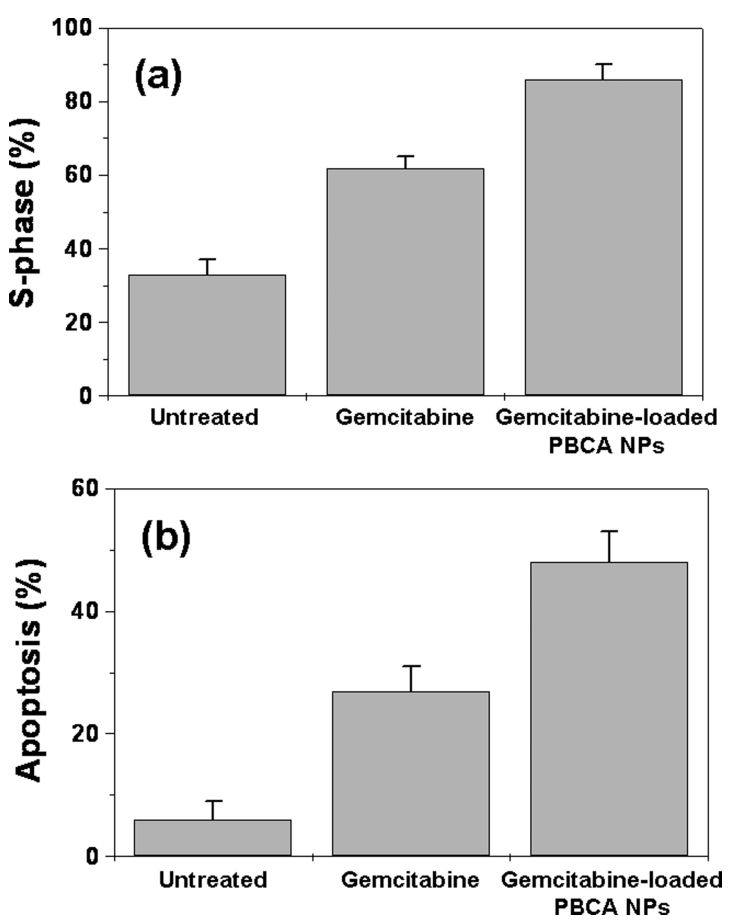

Figure 11. Effect of free gemcitabine $(20 \mathrm{mg} / \mathrm{kg})$ and of gemcitabineloaded PBCA NPs (20 mg/kg equiv) on the L1210 wt leukemia cells collected from tumor bearing mice at day 3 postintravenous injection. (a) S-phase arrest and (b) apoptosis.

This very low tumor cell density suggests the antitumor efficacy of gemcitabine-loaded PBCA NPs as compared with free gemcitabine.

3.7. S-Phase Accumulation and Apoptosis Induction In Vivo. The mechanism of action of gemcitabine involves the incorporation of its active triphosphate form into the replicating DNA and hence shows specificity for the S-phase cells, resulting in cell death either by induction of apoptosis or by mitotic catastrophe in the cells that are unable to conduct apoptosis. $5,36,37$ However, after the drug removal from the cell, especially at sublethal concentrations, the cells may be capable of recovering from the S-phase and become normalized, showing regular growth. ${ }^{38}$ In such cases, the cellular retention of the drug and its time of action could be of high importance. As apparent from 
Figure 11, cells collected from the gemcitabine-loaded PBCA NPs-treated group showed greater S-phase arrest (Figure 11a) and higher apoptosis (Figure 11b) as compared to free gemcitabine-treated mice. Hence, gemcitabine-loaded PBCA NPs could induce S-phase accumulation of the cells, followed by apoptosis indicating their lethal intracellular concentrations. A great and prolonged cellular retention of gemcitabine-loaded PBCA NPs and a dramatic apoptotic induction capability could be hypothesized. In contrast to gemcitabine-loaded PBCA NPs, free gemcitabine induced S-phase arrest and apoptosis but its action was lost rapidly leading to recovery of the cells.

\section{Conclusions}

We have reported the optimal preparation conditions required to obtain gemcitabine-loaded PBCA NPs suitable for parenteral administration. Compared to gemcitabine surface adsorption, the incorporation of this chemotherapy agent into PBCA NPs during the polymerization process has resulted in a greater drug loading and a slower drug release profile. The preclinical results described here, both in vitro and in vivo, demonstrate the efficacy of gemcitabine-loaded PBCA NPs in the treatment of aggressive leukemia L1210 wt subcutaneous tumors. Consequently, gemcitabine loading to PBCA NPs opens interesting perspectives to improve the administration of this chemotherapy compound.

Acknowledgment. Butylcyanoacrylate monomer from Henkel Loctite (Ireland) is gratefully acknowledged. Financial support from Projects PE2005-FQM-410 and PE2008-FQM3993 (Junta de Andalucía) is acknowledged.

\section{References and Notes}

(1) Arias, J. L. Molecules 2008, 13, 2340-2369.

(2) Wong, H. L.; Bendayan, R.; Rauth, A. M.; Li, Y.; Wu, X. Y. Adv. Drug Delivery Rev. 2007, 59, 491-504.

(3) Reddy, L. H. J. Pharm. Pharmacol. 2005, 57, 1231-1242.

(4) Matsuda, A.; Sasaki, T. Cancer Sci. 2004, 95, 105-111.

(5) Reddy, L. H.; Couvreur, P. Curr. Pharm. Des. 2008, 14, 1124-1137.

(6) Storniolo, A. M.; Allerheiligen, S. R. B.; Pearce, H. L. Semin. Oncol. 1997, 24, S7-2S7-7.

(7) Arias, J. L.; Gallardo, V.; Ruiz, M. A.; Delgado, A. V. Int. J. Pharm. 2007, 337, 282-290.

(8) Arias, J. L.; Ruiz, M. A.; López-Viota, M.; Delgado, A. V. Colloids Surf., B 2008, 62, 64-70.

(9) Vauthier, C.; Dubernet, C.; Chauvierre, C.; Brigger, I.; Couvreur, P. J. Controlled Release 2003, 93, 151-160.

(10) Vauthier, C.; Dubernet, C.; Fattal, E.; Pinto-Alphandary, H.; Couvreur, P. Adv. Drug Delivery Rev. 2003, 55, 519-548.

(11) Gelperina, S. E.; Khalansky, A. S.; Skidan, I. N.; Smirnova, Z. S.; Bobruskin, A. I.; Severin, S. E.; Turowski, B.; Zanella, F. E.; Kreuter, J. Toxicol. Lett. 2002, 126, 131-141.
(12) Lherm, C.; Müller, R. H.; Puisieux, F.; Couvreur, P. Int. J. Pharm. 1992, 84, 13-22.

(13) Simeonova, M.; Chorbadjiev, K.; Antcheva, M. Biomaterials 1998, 19, 2187-2193.

(14) Merle, P.; Si-Ahmed, S.; Habersetzer, F.; Abergel, A.; Taieb, J.; Bonyhay, L.; Constantini, D.; Dufour-Lamartinie, J.; Trépo, C. J. Clin. Virol. 2006, 36, S179.

(15) Alyaudtin, R. N.; Reichel, A.; Lobenberg, R.; Ramge, P.; Kreuter, J.; Begley, D. J. J. Drug Targeting 2001, 9, 209-221.

(16) Barraud, L.; Merle, P.; Soma, E.; Lefrançois, L.; Guerret, S.; Chevallier, M.; Dubernet, C.; Couvreur, P.; Trépo, C.; Vitvitski, L. J. Hepatol. 2005, 42, 736-743.

(17) Reddy, L. H.; Murthy, R. S. R. Biomed. Pap. 2004, 148, 161-166.

(18) Vauthier, C.; Labarre, D.; Ponchel, G. J. Drug Targeting 2007, 15, 641-663.

(19) Brigger, I.; Dubernet, C.; Couvreur, P. Adv. Drug Delivery Rev. 2002, 54, 631-651.

(20) Fawaz, F.; Guyot, M.; Lagueny, A. M.; Devissaguet, J. Ph. Int. J. Pharm. 1997, 154, 191-203.

(21) Müller, R. H.; Lherm, C.; Herbort, J.; Couvreur, P. Colloid Polym. Sci. 1991, 269, 147-152.

(22) Sullivan, C. O.; Birkinshaw, C. Biomaterials 2004, 25, 4375-4382.

(23) Wang, C.; He, C.; Tong, Z.; Liu, X.; Ren, B.; Zeng, F. Int. J. Pharm. 2006, 308, 160-167.

(24) Llovet, M. I.; Egea, M. A.; Valero, J.; Alsina, M. A.; García, M. L.; Chauvet, A. Drug Dev. Ind. Pharm. 1995, 21, 1761-1771.

(25) Stolnik, S.; Illum, L.; Davis, S. S. Adv. Drug Delivery Rev. 1995, 16, 195-214.

(26) Brigger, I.; Morizet, J.; Laudani, L.; Aubert, G.; Appel, M.; Velasco, V.; Terrier-Lacombe, M. J.; Desmaële, D.; d'Angelo, J.; Couvreur, P.; Vassal, G. J. Controlled Release 2004, 100, 29-40.

(27) Arias, J. L.; Gallardo, V.; Gómez-Lopera, S. A.; Delgado, A. V. J. Biomed. Nanotechnol. 2005, 1, 214-223.

(28) Konerding, D.; James, T. L.; Trump, E.; Soto, A. M.; Marky, L. A.; Gmeiner, W. H. Biochemistry 2008, 41, 839-846.

(29) Silverstein, R. M.; Webster, F. X. In Spectrometric Identification of Organic Compounds, 6th ed.; Silverstein, R. M., Webster, F. X., Eds.; John Wiley \& Sons: New York, 1998; pp 144-249.

(30) McCarron, P. A.; Woolfson, A. D.; Keating, S. M. J. Pharm. Pharmacol. 2000, 52, 1451-1459.

(31) Simeonova, M.; Velichkova, R.; Ivanova, G.; Enchev, V.; Abrahams, I. J. Drug Targeting 2004, 12, 49-56.

(32) Arias, J. L.; Gallardo, V.; Linares-Molinero, F.; Delgado, A. V. J. Colloid Interface Sci. 2006, 299, 599-607.

(33) Stella, B.; Arpicco, S.; Rocco, F.; Marsaud, V.; Renoir, J. M.; Cattel, L.; Couvreur, P. Int. J. Pharm. 2007, 344, 71-77.

(34) Arias, J. L.; Gallardo, V.; Gómez-Lopera, S. A.; Plaza, R. C.; Delgado, A. V. J. Controlled Release 2001, 77, 309-321.

(35) Sullivan, C. O.; Birkinshaw, C. Polym. Degrad. Stab. 2002, 78, 715.

(36) Huang, P.; Chubb, S.; Hertel, L. W.; Grindey, G. B.; Plunkett, W. Cancer Res. 1991, 51, 6110-6117.

(37) Huang, B.; Plunkett, W. Cancer Chemother. Pharmacol. 1995, 36, $181-188$.

(38) Shi, Z.; Azuma, A.; Sampath, D.; Li, Y. X.; Huang, P.; Plunkett, W. Cancer Res. 2001, 61, 1065-1072.

BM900644A 